\title{
Nationwide incidence and risk factors for posttraumatic seizures in children with traumatic brain injury
}

\author{
Kavelin Rumalla, BA, ${ }^{1}$ Kyle A. Smith, MD, ${ }^{2}$ Vijay Letchuman, ${ }^{1}$ Mrudula Gandham,, \\ Rachana Kombathula, ${ }^{1}$ and Paul M. Arnold, MD ${ }^{2}$
}

${ }^{1}$ School of Medicine, University of Missouri-Kansas City, Missouri; and ${ }^{2}$ Department of Neurosurgery, University of Kansas Medical Center, Kansas City, Kansas

OBJECTIVE Posttraumatic seizures (PTSs) are the most common complication following a traumatic brain injury (TBI) and may lead to posttraumatic epilepsy. PTS is well described in the adult literature but has not been studied extensively in children. Here, the authors utilized the largest nationwide registry of pediatric hospitalizations to report the national incidence, risk factors, and outcomes associated with PTS in pediatric TBI.

METHODS The authors queried the Kids' Inpatient Database (KID) using ICD-9-CM codes to identify all patients (age $<21$ years) who had a primary diagnosis of TBI (850.xx-854.xx) and a secondary diagnosis of PTS $(780.33,780.39)$. Parameters of interest included patient demographics, preexisting comorbidities, hospital characteristics, nature of injury (open/closed), injury type (concussion, laceration/contusion, subarachnoid hemorrhage, subdural hematoma, or epidural hematoma), loss of consciousness (LOC), surgical management (Clinical Classification Software code 1 or 2), discharge disposition, in-hospital complications, and in-hospital mortality. The authors utilized the IBM SPSS statistical package (version 24) for univariate comparisons, as well as the identification of independent risk factors for PTS in multivariable analysis (alpha set at <0.05).

RESULTS The rate of PTS was $6.9 \%$ among 124,444 unique patients hospitalized for TBI. The utilization rate of continuous electroencephalography (cEEG) was $0.3 \%$ and increased between $2003(0.1 \%)$ and $2012(0.7 \%)$. The most common etiologies of TBI were motor vehicle accident $(n=50,615)$, accidental fall $(n=30,847)$, and blunt trauma $(n=$ 13,831). However, the groups with the highest rate of PTS were shaken infant syndrome $(41.4 \%)$, accidental falls $(8.1 \%)$, and cycling accidents (7.4\%). In multivariable analysis, risk factors for PTS included age 0-5 years (compared with 6-10, 11-15, and 16-20 years), African American race (OR 1.4), $\geq 3$ preexisting comorbidities (OR 4.0), shaken infant syndrome (OR 4.4), subdural hematoma (OR 1.6), closed-type injury (OR 2.3), brief LOC (OR 1.4), moderate LOC (OR 1.5), and prolonged LOC with baseline return (OR 1.8). Surgically managed patients were more likely to experience PTS (OR 1.5) unless they were treated within 24 hours of admission (OR 0.8). PTS was associated with an increased likelihood of in-hospital complications (OR 1.7) and adverse (nonroutine) discharge disposition (OR 1.2), but not in-hospital mortality (OR 0.5). The overall utilization rate of cEEG was $1.3 \%$ in PTS patients compared with $0.2 \%$ in patients without PTS. Continuous EEG monitoring was associated with higher rates of diagnosed PTS ( $35.4 \%$ vs $6.8 \%$; OR 4.9, $p<0.001)$.

CONCLUSIONS PTS is common in children with TBI and is associated with adverse outcomes. Independent risk factors for PTS include younger age (< 5 years), African American race, increased preexisting comorbidity, prolonged LOC, and injury pattern involving cortical exposure to blood products. However, patients who undergo urgent surgical evacuation are less likely to develop PTS.

https://thejns.org/doi/abs/10.3171/2018.6.PEDS1813

KEYWORDS posttraumatic seizure; traumatic brain injury; trauma

ABBREVIATIONS AED = antiepileptic drug; $A H R Q=$ Agency for Healthcare Research and Quality; CCS = Clinical Classification Software; cEEG = continuous electroencephalography; EDH = epidural hematoma; HCUP = Healthcare Cost and Utilization Project; ICD-9-CM = International Classification of Diseases, Ninth Revision, Clinical Modification; KID = Kids' Inpatient Database; LOC = loss of consciousness; PTS = posttraumatic seizure; SAH = subarachnoid hemorrhage; SDH = subdural hematoma; $\mathrm{TBI}=$ traumatic brain injury.

SUBMITTED January 8, 2018. ACCEPTED June 25, 2018.

INCLUDE WHEN CITING Published online September 21, 2018; DOI: 10.3171/2018.6.PEDS1813. 
$\mathrm{P}$ OSTTRAUMATIC seizures (PTSs) are one of the most common complications following a traumatic brain injury (TBI) in adults. ${ }^{2,17}$ Seizures that occur within 24 hours after the incident are categorized as immediate PTSs. Seizures that occur within a week of the incident are termed early, and seizures within a month are categorized as late PTSs. ${ }^{1}$ Prophylactic antiepileptic drugs (AEDs) are routinely administered at some institutions, but the indications and protocols vary significantly. Intervention for PTS is justified by evidence that early seizures following TBI can hinder cerebral recovery through several mechanisms, including increased intracranial pressure and hypoxic-ischemic injury. ${ }^{9,27}$ PTS is also one of the early signs of developing posttraumatic epilepsy. While PTS is well studied in adults, the causes of TBI in children as well as the resulting injury are inherently unique, even among pediatric age groups. The existing epidemiological literature concerning the rates, causes, and risk factors for pediatric PTS is relatively scarce and consists of a handful of single-center institutional experiences. Here, we utilized the largest database of pediatric hospital stays in the US to establish benchmark rates of PTS for future investigation and heighten awareness of this deleterious complication. Furthermore, we attempt to identify nonmodifiable risk factors to guide risk stratification (e.g., etiology of PTS, clinical presentation) and modifiable risk factors to inform clinical decision-making (e.g., continuous electroencephalography [cEEG], surgical intervention) and future research designs. We chose a large administrative data set because the true rate of PTS in children is unclear and complex stratified analyses require ample statistical power. Lastly, while not the primary scope of our paper, we sought to explore several controversial topics in the literature, including management trends over time, cEEG monitoring, and surgical intervention.

\section{Methods \\ Data Source}

The Kids' Inpatient Database (KID), a component of the Healthcare Cost and Utilization Project (HCUP), is a national database that is maintained under the Agency for Healthcare Research and Quality (AHRQ). ${ }^{14}$ It is released every 3 years $(2003,2006,2009$, and 2012). We used this database to analyze trends in hospital characteristics, outcomes, quality, and costs of approximately 7 million discharge records for patients who were younger than 21 years from 22 states. The KID allows researchers to look at estimates of population attributes such as patient and hospital demographics with an accepted level of accuracy due to the immense size of the sample. The database contains 1 primary diagnosis/procedure field and $15 \mathrm{sec}-$ ondary diagnosis/procedure fields. The fields are searched using International Classification of Diseases, Ninth Revision, Clinical Modification (ICD-9-CM) codes. The AHRQ's Clinical Classification Software (CCS) groups ICD-9-CM codes into clinically meaningful categories that can be used alternatively to search for diagnoses of lower specificity.$^{13}$ More information regarding the KID can be found at https://www.hcup-us.ahrq.gov/db/nation/ kid/kiddbdocumentation.jsp. All authors with access to the KID data have read and completed the online HCUP Data Use Agreement Training and signed the appropriate documents. In agreement with the HCUP Data Use Agreement, no sample size smaller than 11 was reported.

\section{Inclusion and Exclusion Criteria}

We queried the KID to identify all patients with a primary diagnosis of TBI, which included cases of concussion $(850.0-850.5,850.9)$, cerebral laceration/contusion (851.01-), subarachnoid hemorrhage (SAH; 852.0X, 852.1X), subdural hematoma (SDH; 852.2X, 852.3X), epidural hematoma (EDH; 852.4X, 852.5X), and other/ unspecified (853.XX, 854.XX). We differentiated between open and closed intracranial injuries using the 4th digit of the ICD-9-CM code and used the 5th digit to grade the patient level of consciousness at admission. The database was then searched for a secondary diagnosis code for posttraumatic seizures $(780.33,780.39)$.

\section{Predictor Variables}

We abstracted and defined variables pertaining to patient demographics, preexisting medical comorbidities, admission characteristics, hospital characteristics, etiology of TBI (E codes), indication for surgery (type of TBI, open/closed wound, severity of TBI), and surgical management (CCS code 1 or 2). The patient demographic variables included categorical age $(0-5,6-10,11-15,16-20$ years), sex (male or female), and median household income (0-25th, 26th-50th, 51st-75th, 76th-100th percentiles). The overall preexisting comorbidity per patient was assessed via the Elixhauser Comorbidity Index, which was computed using AHRQ software and consists of a list of 31 preexisting comorbidities that are known to be strongly associated with morbidity and mortality at discharge. ${ }^{10}$ Admission characteristics included admission type (nonelective vs elective) and day of admission (weekday vs weekend). Hospital characteristics included hospital bedsize (small, medium, large) and hospital type (metropolitan nonteaching, metropolitan teaching, nonmetropolitan). Management characteristics included cEEG monitoring (ICD-9-CM code 89.19) and surgical intervention.

\section{Outcome Variables}

We also assessed the impact of PTS on in-hospital complication rate, length of stay, total costs, discharge disposition, and in-hospital mortality rates. Complication variables were defined using ICD-9-CM secondary diagnosis fields: respiratory $(518.4,518.5,518.81-518.84)$, cardiac (997.1 and 410), renal and urinary (584 and 997.5), hematoma/hemorrhage (998.1-998.13), neurological (997.00-997.09), thromboembolic (415, 415.11-415.19, 451.0-451.9), and septicemia (998.51, 998.59, 996.63, 996.67). The total hospital charge variables in the KID do not accurately represent how much hospitals receive in payment so HCUP provides cost-to-charge ratio files that are used to convert "charges" to "costs."

\section{Statistical Analysis}

The HCUP KID outlines the data management steps that must be carried out to create the original data set for 
statistical analysis. For this study, IBM SPSS (version 24, IBM Corp.) was used for all analyses, and statistical significance was set at an alpha of $<0.05$. Descriptive statistics were collected for all patients in the defined cohort, and the incidence rates of PTS were collected. Additional stratified analysis was performed by various categorical variables to determine which groups had the highest rates of PTS. Univariate analysis was performed to identify risk factors associated with PTS. The Pearson chi-square test was used for categorical variables and the independent samples t-test for continuous variables. Next, we designed multivariable models to identify risk factors independently associated with PTS. Variables with $\mathrm{p}<0.1$ were included in the multivariable analysis, and factors with $p<0.05$ were retained in the model. The effect of PTS on in-hospital complications, discharge disposition, and in-hospital mortality was explored in univariate and multivariable analyses using the same methodology described above.

\section{Results \\ Study Population}

We identified a total of 124,444 pediatric patients who were hospitalized for TBI in 2003, 2006, 2009, and 2012. The mean age of the cohort was $12.0 \pm 6.7,31.1 \%$ were female, and $19.3 \%$ had at least 1 preexisting comorbidity. Overall, 95.8\% of patients were admitted at hospitals in an urban location, $69.8 \%$ at large-bedsize hospitals, and $82.5 \%$ at teaching hospitals (Table 1). The most common etiologies leading to TBI were motor vehicle accident (n $=50,615)$, accidental fall $(\mathrm{n}=30,847)$, and blunt trauma $(\mathrm{n}=13,831)$. The most common type of TBI was concussion $(29.0 \%)$, followed by contusion $(n=11,661)$ and SDH $(\mathrm{n}=10,249)$. The rate of surgical management was $9.7 \%$ and remained relatively steady from 2003 to 2012 (10.2\%, $9.6 \%, 9.3 \%$, and 9.8\%; Table 2). The average length of stay was 4.8 days, and the average cost of hospitalization was $\$ 17,485$. The overall complication rate was $12.7 \%$, and pulmonary complications were the most prevalent at $12.1 \%$. The rate of adverse discharge (not routine) was $18.4 \%$, and the in-hospital mortality rate was $5.4 \%$.

\section{Incidence and Risk Factors for PTS}

The clinical rate of PTS in this population was 6.9\% and increased to $35.4 \%$ in patients who underwent cEEG monitoring. The rate of PTS decreased from $7.6 \%$ in 2003 to $6.2 \%$ in 2012 ( $p<0.001$, Fig. 1). Patient factors such as young age, increased comorbidity, and racial group influenced PTS rates (Table 1). Etiologies with the highest rate of PTS (but not necessarily the most common overall) were shaken infant syndrome (41.4\%), accidental falls (8.1\%), and cycling accidents (7.4\%) (Table 2 and Fig. 2). In subgroup analysis of the youngest cohort ( $0-5$ years), we found that the rate of PTS was $40.8 \%(n=119)$ in victims of shaken infant syndrome compared with $9.7 \%$ ( $\mathrm{n}=$ $2,789)$ in the remaining TBI patients $(\mathrm{p}<0.001)$. However, removing shaken infant syndrome from the analysis only reduced the overall PTS rate in this cohort from $10 \%$ to 9.7\%. Patients with SDH and increased duration of loss of consciousness (LOC) also had higher rates of PTS (Table 2 and Fig. 3).
TABLE 1. Patient demographics and hospital characteristics of 124,444 patients with TBI in the KID

\begin{tabular}{|c|c|c|c|}
\hline Characteristic & No. of Patients & No. w/ PTS (\%) & $p$ Value \\
\hline Total no. & 124,444 & $8,597(6.9)$ & \\
\hline Age group, yrs & & & $<0.001$ \\
\hline $0-5$ & 29,045 & $2,914(10.0)$ & \\
\hline $6-10$ & 14,816 & $850(5.7)$ & \\
\hline $11-15$ & 25,610 & $1,748(6.8)$ & \\
\hline $16-20$ & 54,974 & $3,085(5.6)$ & \\
\hline Sex $(\%)$ & & & 0.76 \\
\hline Female & 38,696 & $2,657(6.9)$ & \\
\hline Male & 85,748 & $5,940(6.9)$ & \\
\hline Race & & & $<0.001$ \\
\hline White & 59,605 & $3,635(6.1)$ & \\
\hline African American & 14,029 & $1,253(9.0)$ & \\
\hline Hispanic & 18,990 & $1,447(7.6)$ & \\
\hline Other & 8,755 & $625(7.1)$ & \\
\hline Median household income & & & 0.06 \\
\hline 0-24th percentile & 32,988 & $2,408(7.3)$ & \\
\hline 25-49th percentile & 30,090 & $2,046(6.8)$ & \\
\hline 50-74th percentile & 29,820 & $2,058(6.9)$ & \\
\hline 75-99th percentile & 27,957 & $1,817(6.5)$ & \\
\hline Hospital location & & & $<0.001$ \\
\hline Urban & 115,627 & $8,046(7.0)$ & \\
\hline Rural & 5,073 & $236(4.7)$ & \\
\hline Hospital bedsize & & & 0.56 \\
\hline Small & 10,189 & $725(7.1)$ & \\
\hline Medium & 26,255 & $1,746(6.7)$ & \\
\hline Large & 84,256 & $5,811(6.9)$ & \\
\hline Hospital teaching status & & & $<0.001$ \\
\hline Nonteaching & 20,250 & $1,151(5.7)$ & \\
\hline Teaching & 95,377 & $6,895(7.2)$ & \\
\hline
\end{tabular}

In multivariable analysis, we identified risk factors independently associated with PTS (Table 3 and Fig. 4). Since cEEG is known to influence PTS rates, cEEG utilization was also adjusted for in multivariable analysis. Younger age ( $0-5$ years) was associated with increased likelihood of PTS (Table 3). Patients of African American descent (OR 1.4) and Hispanic descent (OR 1.2) were slightly more predisposed (both $\mathrm{p}<0.001$ ). Teaching hospitals were more likely to diagnose PTSs (OR 1.1, p = 0.001). Children with high comorbidity burden (3 or more) were more likely to develop PTS (OR 4.0, $\mathrm{p}<0.001)$. The rate of PTS was significantly higher in the unfortunate case of shaken infant syndrome (OR 4.4, $\mathrm{p}<0.001)$. Patients sustaining injury patterns of closed-type (OR 2.3) and SDH (OR 1.6, 95\% CI 1.5-2.3) were more likely to have PTS (all $\mathrm{p}<0.001$ ). Those with EDH were less likely (OR $0.5, \mathrm{p}<0.001)$. Increasing duration of LOC was associated with increased rate of PTS: brief ( $<1$ hour, OR 1.4), moderate (1-24 hours, OR 1.5), and prolonged with baseline return ( $\geq 24$ hours, OR 1.8) (all $\mathrm{p}<0.005)$. Prolonged LOC without return (coma) was not associated with increased PTS risk. 
TABLE 2. Preexisting comorbidities and clinical presentation (severity of TBI)

\begin{tabular}{|c|c|c|c|}
\hline Characteristic & $\begin{array}{l}\text { No. of } \\
\text { Patients }\end{array}$ & $\begin{array}{l}\text { No. w/ PTS } \\
(\%)\end{array}$ & $\begin{array}{c}p \\
\text { Value }\end{array}$ \\
\hline Total no. $(\%)$ & 124,444 & $8,597(6.9)$ & \\
\hline Comorbidity score (\%) & & & $<0.001$ \\
\hline 0 & 89,669 & $4,842(5.4)$ & \\
\hline 1 & 24,014 & $2,233(9.3)$ & \\
\hline 2 & 7,700 & $1,001(13.0)$ & \\
\hline$\geq 3$ & 3,061 & $530(17.3)$ & \\
\hline \multicolumn{4}{|l|}{ Etiology of injury $(\%)$} \\
\hline Shaken infant syndrome & 292 & $121(41.4)$ & $<0.001$ \\
\hline Accidental fall & 30,847 & $2,499(8.1)$ & $<0.001$ \\
\hline Cycling accident & 4,532 & $335(7.4)$ & 0.19 \\
\hline Blunt trauma & 13,831 & $968(7.0)$ & 0.61 \\
\hline MVA & 50,615 & $2,480(4.9)$ & $<0.001$ \\
\hline Firearm & 2,165 & $97(4.5)$ & $<0.001$ \\
\hline Other/unspecified/missing E code & 22,162 & - & - \\
\hline \multicolumn{4}{|l|}{$\begin{array}{l}\text { Consciousness at clinical presenta- } \\
\text { tion }\end{array}$} \\
\hline No LOC & 22,563 & $1,421(6.3)$ & 0.004 \\
\hline Brief LOC (<1 hr) & 17,054 & $1,279(7.5)$ & 0.02 \\
\hline Moderate LOC (1-24 hrs) & 752 & $80(10.6)$ & 0.001 \\
\hline $\begin{array}{l}\text { Prolonged LOC ( } \geq 24 \mathrm{hrs}) \\
\text { baseline return }\end{array}$ & 845 & $117(13.9)$ & $<0.001$ \\
\hline $\begin{array}{l}\text { Prolonged LOC ( } \geq 24 \mathrm{hrs}) \text {, no } \\
\text { return }\end{array}$ & 3,532 & $226(6.4)$ & 0.38 \\
\hline Other & 32,749 & $2,423(7.4)$ & 0.001 \\
\hline \multicolumn{4}{|l|}{ Type of TBI } \\
\hline Concussion & 36,078 & $2,165(6.0)$ & $<0.001$ \\
\hline Cerebral laceration/contusion & 11,661 & $863(7.4)$ & 0.88 \\
\hline $\mathrm{SAH}$ & 7,444 & $484(6.5)$ & 0.26 \\
\hline $\mathrm{SDH}$ & 10,249 & $1,230(12.0)$ & $<0.001$ \\
\hline $\mathrm{EDH}$ & 2,732 & $109(4.0)$ & $<0.001$ \\
\hline Other & 9,329 & $709(7.6)$ & 0.05 \\
\hline Nature of injury & & & $<0.001$ \\
\hline Open & 50 & $2(3.6)$ & \\
\hline Closed & 347 & $24(6.9)$ & \\
\hline cEEG monitoring & & & $<0.001$ \\
\hline No & 115,644 & $231(0.2)$ & \\
\hline Yes & 8,486 & $110(1.3)$ & \\
\hline Surgical evacuation & & & $<0.001$ \\
\hline No & 111,362 & $7,239(6.5)$ & \\
\hline Yes & 12,082 & $1,293(10.7)$ & \\
\hline Evacuation on admission & & & $<0.001$ \\
\hline No & 117,295 & $7,859(6.7)$ & \\
\hline Yes & 7,150 & $686(9.6)$ & \\
\hline
\end{tabular}

MVA = motor vehicle accident; $-=$ due to missing variables in original KID data, statistics cannot be calculated.

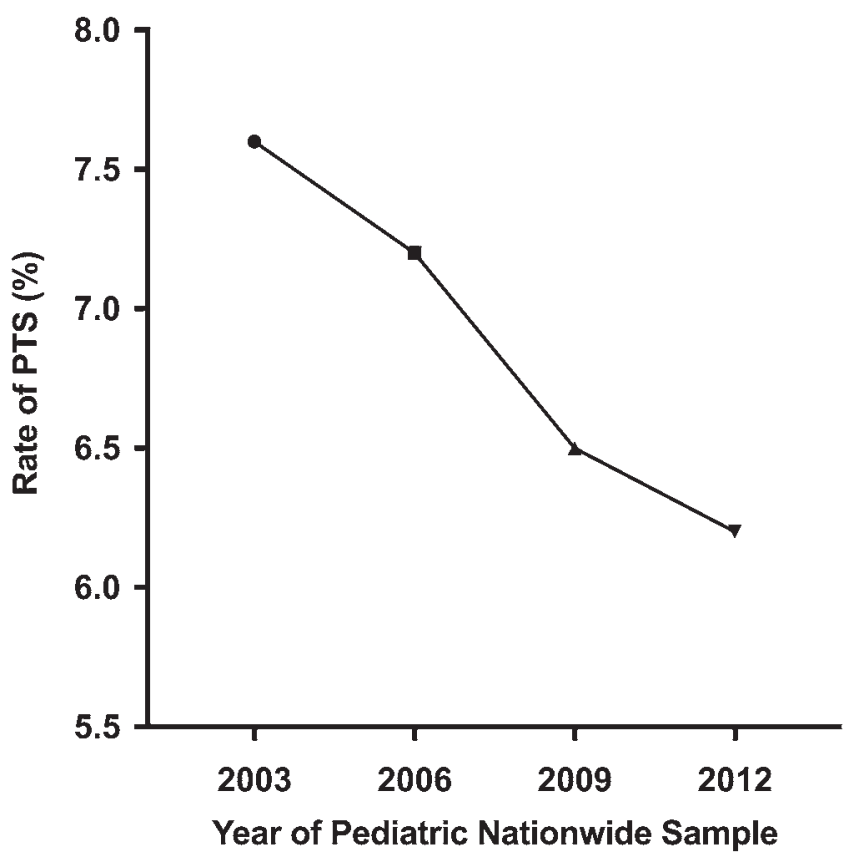

FIG. 1. Trends in rate of pediatric PTS over time.

\section{Continuous EEG Monitoring and Surgical Intervention}

Continuous EEG was utilized in $0.3 \%$ of TBI patients and this rate increased from $0.1 \%$ in 2003 to $0.7 \%$ in 2012. Continuous EEG utilization was significantly more common in certain groups, including cases of shaken infant syndrome $(3.3 \%$ vs $0.2 \%, \mathrm{p}<0.001)$, younger age $(0-5$ years, $0.6 \%$; $6-10$ years, $0.2 \%$; $11-15$ years, $0.2 \%$; and $16-$ 20 years $0.1 \% ; \mathrm{p}<0.001)$, and higher comorbidity score $(0$, $0.1 \% ; 1,0.4 \% ; 2,0.7 \%$; and $3,1.1 \% ; \mathrm{p}<0.001)$. The utilization rate was higher in teaching hospitals $(0.3 \%$ vs $0.0 \%$ in nonteaching, $\mathrm{p}<0.001)$ and urban hospitals $(0.3 \%$ vs $0.0 \%$ in rural, $\mathrm{p}<0.001)$. Patients with prolonged LOC with baseline return (0.6\% vs $0.3 \%)$ and without baseline return $(0.5 \%$ vs $0.2 \%)$ were the most likely to receive cEEG monitoring (both $\mathrm{p}<0.001)$. Patients with SAH $(0.5 \%$ vs $0.2 \%)$ and $\mathrm{SDH}(0.6 \%$ vs $0.2 \%)$ were significantly more likely to receive monitoring $(\mathrm{p}<0.001)$.

The overall rate of cEEG usage was $1.3 \%$ in patients with PTS compared with $0.2 \%$ without PTS. The rate of PTS was $35.4 \%$ in the cEEG cohort compared with $6.8 \%$ in the non-cEEG cohort. In multivariable analysis, utilization of cEEG monitoring was associated with significantly higher rates of diagnosed PTS (OR 4.9, p < 0.001). Overall, surgical management was associated with higher rate of PTS (OR 1.5, p < 0.001). However, surgery performed within 24 hours of admission was associated with decreased rate of PTS (OR 0.8, $\mathrm{p}=0.01$ ).

\section{Outcomes of Posttraumatic Seizure}

Patients suffering 1 or more in-hospital complications had significantly higher rates of PTSs (12.2\% vs 6.9\%, p $<0.001)$. The rate of PTSs was greater in patients transferred to short-term hospitals (12.0\%), specialized nursing facilities or intermediate care facilities (11.1\%), or home healthcare $(9.2 \%)$ compared with those discharged 


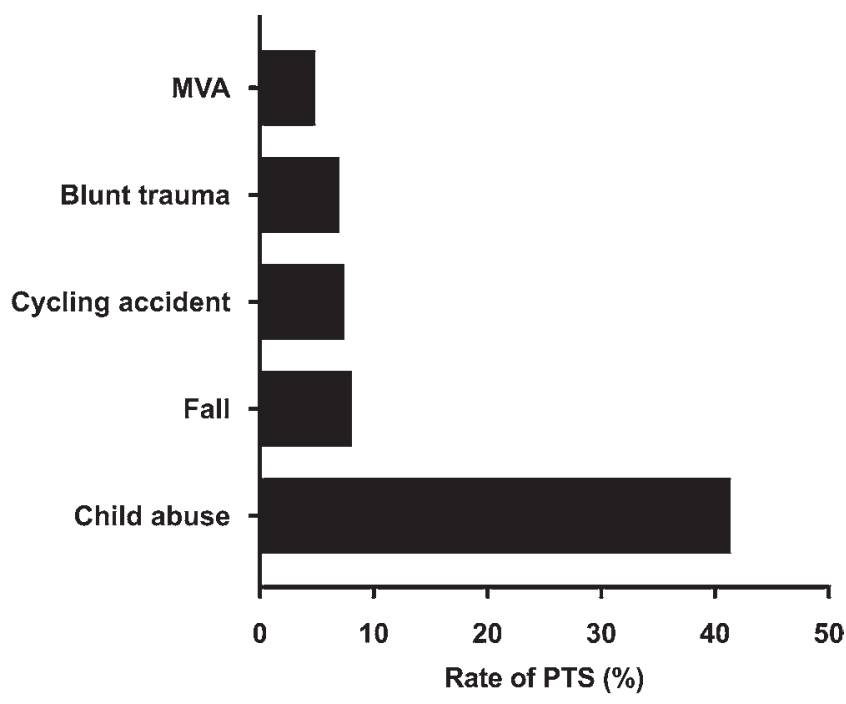

FIG. 2. Effect of TBI etiology on the rate of PTS. MVA = motor vehicle accident.

routinely $(6.5 \%)(\mathrm{p}<0.001)$. However, the rate of PTS was lower among patients suffering in-hospital mortality (4.3\% vs $6.5 \%)$. In multivariable analysis, PTS was associated with increased likelihood of in-hospital complication occurrence (OR 1.65, 95\% CI 1.54-1.78, p < 0.001) and adverse (not routine) discharge disposition (OR 1.16, 95\% CI 1.07-1.26, $\mathrm{p}<0.001)$, but decreased likelihood of inhospital mortality (OR $0.47,95 \%$ CI $0.40-0.55$, p < 0.001 ). The complete analysis of in-hospital complications and discharge disposition is shown in Table 4.

\section{Discussion}

We report the first national incidence of PTS (6.9\%) in a cohort of 124,444 pediatric patients with TBI, which is consistent with the lower end of the range described in previous single-center series $(6 \%-19 \%))^{4,15}$ However, the rate of PTS was much higher (35.4\%) in patients who underwent cEEG, which is similar to the rate in an earlier study (30\%). ${ }^{21}$ The rates of other complications following TBI in adult populations have been reported in the literature, including hydrocephalus $(0.7 \%-45 \%),{ }^{8,19,25,29}$ stroke $(0.6 \%),{ }^{20} \mathrm{CSF}$ leakage $(0.1 \%),{ }^{20}$ and neurological infections $(0.6 \%){ }^{20}$ Hydrocephalus was much less common than PTS in pediatric TBI. ${ }^{20,25}$

Several prior single-institution studies have investigated the rate of PTS in children with TBI. Hale et al. performed a retrospective analysis of 1,814 children at a single center to identify risk factors for postdischarge persistent seizures, which could not be evaluated in the present study. ${ }^{12}$ They identified bilateral bleeding, body temperature instability, and SDH as predictors of postdischarge seizure activity. Liesemer et al. studied early PTS in a retrospective observational study of 299 children admitted with moderate to severe TBI at a level 1 trauma center. ${ }^{18}$ They reported a PTS rate of $12 \%$, which is similar to our reported rates in several cohorts: SDH (12.0\%), moderate LOC (10.6\%), and prolonged LOC with baseline return (13.9\%) cohorts. Dingman et al. analyzed retrospec-

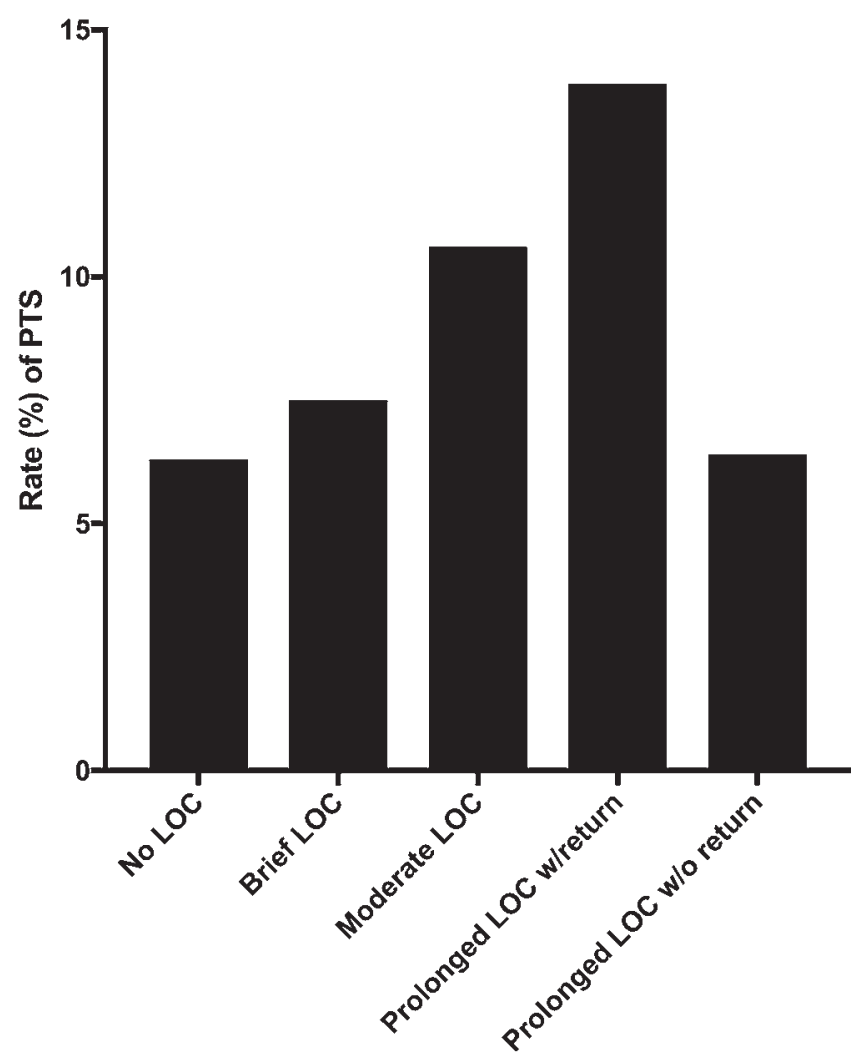

FIG. 3. Effect of loss of consciousness on rate of PTS.

tive data of 58 children who underwent cEEG monitoring following moderate to severe shaken infant syndrome and identified a seizure rate of $51.2 \% .{ }^{9}$ We found a PTS rate of $41.4 \%$ in shaken infant syndrome with only a minority of patients receiving cEEG monitoring. Huguenard et al. analyzed a single-institution cohort of patients with mild TBI, and their results suggested a low PTS rate of about $6.8 \%$ (per EEG). Patients with negative findings on EEG were very unlikely to be prescribed AEDs, but only $66.7 \%$ of patients with abnormal EEG findings were prescribed AEDs..$^{15}$ O'Neill et al. performed a single-institution retrospective analysis of 144 children with TBI (102 with moderate/severe TBI) who underwent cEEG. They reported a PTS rate of $30 \%$ in which $40 \%$ of cases were detected subclinically via cEEG. ${ }^{21}$

\section{Time Trends and Etiologies of PTS}

The decrease in PTS rate between 2003 and 2012 was accompanied by constant rates of surgical management, increased utilization of routine AEDs, and increased frequency of routine cEEG (0.1\% in 2003 and $0.7 \%$ in 2012). The true rate of seizure activity in TBI patients is likely much higher than diagnosed, since cEEG is underutilized, particularly in patients having subclinical seizures when comatose. ${ }^{21}$ There is variability in the reported rate of PTS due to bias concerning cEEG, since academic centers are more likely to have higher rates of cEEG utilization and thus higher sensitivity for detection of PTS compared with nationwide rates. The uptrend in utilization is likely due to increased awareness of PTS and rapidly evolving and 


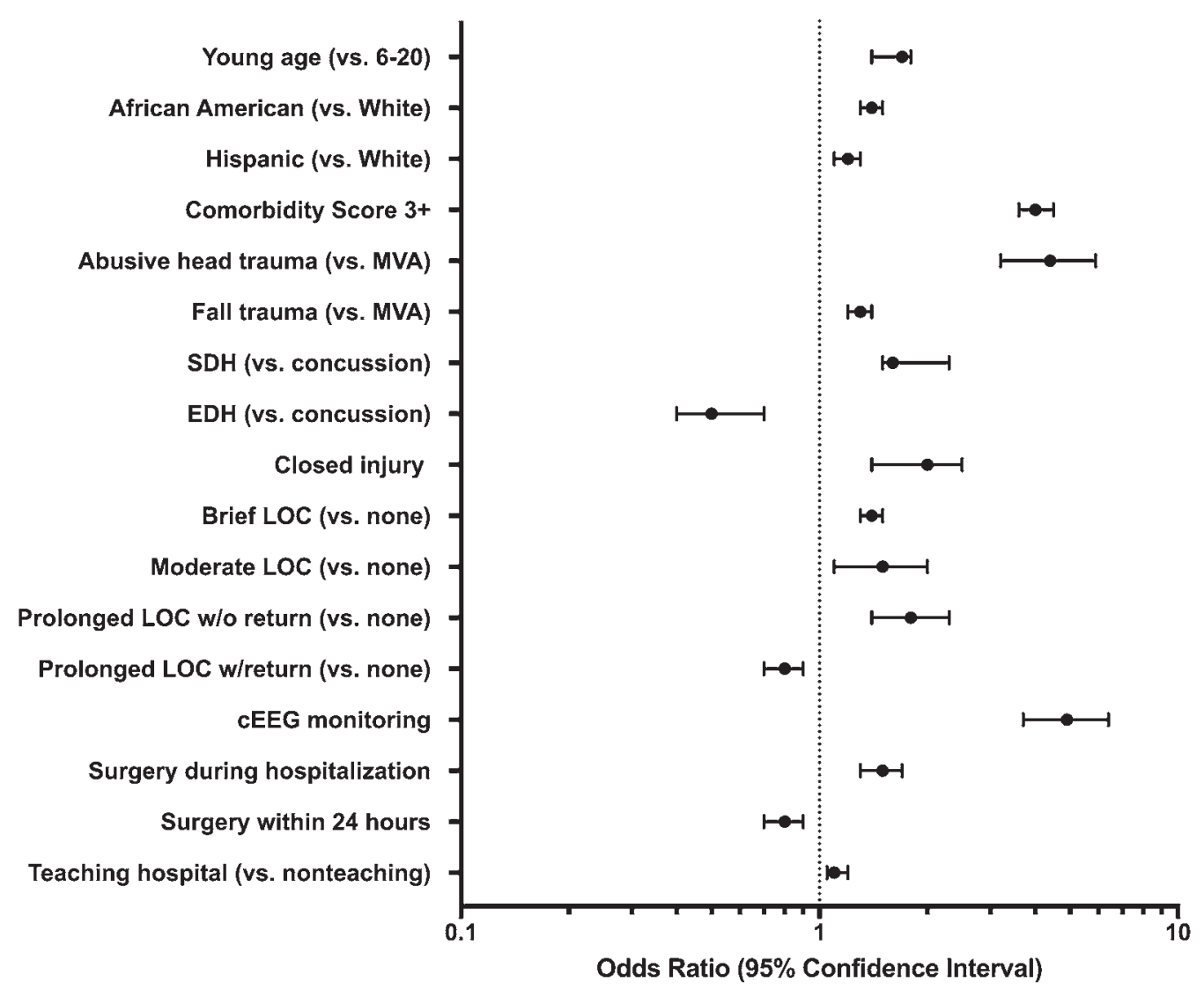

FIG. 4. Multivariable predictors of PTS.

accessible technology outside of major academic hospitals. ${ }^{27}$ We found that teaching hospitals were associated with very slight (10\%) increased risk of PTS, likely due to increased utilization of cEEG.

\section{Modifiable Risk Factors for PTS}

Our results suggest that surgical intervention and cEEG are 2 potentially modifiable risk factors for PTS. The utilization of AEDs is also usually considered in this category but could not be evaluated in this study, as the ICD-9-CM coding system is unable to identify the presence or absence of most pharmaceutical drugs.

Urgent surgical intervention ( $<24$ hours) was slightly protective (20\% decreased likelihood) against PTS, presumably due to reduced duration of LOC and mass effect. The urgent intervention should theoretically reduce epileptogenesis by reducing prolonged compression and removing epileptogenic hemoglobin degradation products from the cascade, leading to epileptogenesis. ${ }^{11,30}$ The beneficial effect of urgent surgical intervention in these patients likely varies based on the overall risk profile. Further prospective study is advised to evaluate the causal relationship, including identification of the most ideal time to surgery, risk groups that benefited the most, and whether the statistical difference is clinically significant.

The rate of cEEG utilization in our study was only $0.3 \%$, which is similar to that in a prior pediatric report $(<1 \%) .{ }^{15}$ Continuous EEG was a predictor of increased PTS rate, which supports the theory of increased subclinical seizure detection. We observed a paradoxical trend of increased PTS rates as the duration of temporary LOC increased (independent, multivariable), which may seem counterintuitive considering the low rate of cEEG. However, since permanent LOC was not associated with increased PTS and the overall cEEG rate is low $(0.3 \%)$, we can safely assume that seizures were clinically detected pre- or post-LOC. In patients in a state of persistent coma, we did not find an increased risk for PTS, which is consistent with that reported in previous studies, demonstrating an inverse correlation between Glasgow Coma Scale score and PTS risk. ${ }^{4}$

The utility of cEEG in PTS management depends on whether a given institution is readily prescribing AEDs as prophylaxis. Previous studies have found that cEEG does frequently lead to changes in seizure management (50\%), including withdrawal of AEDs. ${ }^{9,16}$ Thus, if a decision is made to not prescribe AEDs, it is essential to monitor for PTS using cEEG.

Regarding AEDs, prior studies in adults have investigated the comparative efficacy of several agents in the prevention and/or suppression of PTS. Liesemer et al. reported a PTS rate of $12 \%$ in patients with moderate to severe TBI which was initially $68 \%$ within the first 12 hours before AEDs were administered..$^{18}$ A recent review of the literature revealed that while phenytoin and carbamazepine are suspected to decrease about $25 \%$ of posttraumatic seizures, the small sample sizes in the prior studies were 
not powered to detect less than a $50 \%$ reduction. ${ }^{28} \mathrm{~A}$ systematic review suggested that levetiracetam is preferred over phenytoin for the prevention of late posttraumatic seizures. ${ }^{6}$ A recent study of severe pediatric TBI by Ruzas et al. investigated the rates of EEG and AED utilization. ${ }^{26}$ They found that children with risk factors for PTS, including shaken infant syndrome, $\mathrm{SDH}$, and young age, were more likely to receive monitoring for PTS, but that rate of AED prophylaxis exceeded the rate of EEG monitoring.

\section{Nonmodifiable Risk Factors for PTS}

In an effort to provide data to guide early risk stratification, we identified nonmodifiable risk factors at initial clinical presentation independently associated with pediatric PTS. These risk factors include younger age, racial group, comorbidity, etiology of TBI, severity of TBI, and type of TBI. The association between younger age and increased PTS risk has been reported previously ${ }^{4,18}$ and is attributed to the structural and functional immaturity of the brain at younger ages. ${ }^{3-5,23}$ However, our study provides a stratified analysis of PTS rates by age brackets with a large enough sample size to allow for generalizability. In a subgroup analysis of the youngest cohort ( $0-5$ years old), we found that shaken infant syndrome had higher rates of PTS, but only accounted for $10 \%$ of young patients with PTS. Removing shaken infant syndrome victims from the subgroup of young children only reduced the overall PTS rate from $10.0 \%$ to $9.7 \%$. African American and Hispanic children were also more predisposed, possibly due to socioeconomic inequality and less access to hospitals with routine AED administration, cEEG, and surgical intervention. The disparity related to racial groups may also be related to pattern of injury, environmental factors, ${ }^{24}$ and genetics. ${ }^{24}$ As expected, a high preexisting comorbidity score was also a risk factor.

The etiology of injury, type of TBI, and duration of LOC significantly affected PTS rates. Our conclusions were drawn from multivariable analyses attempting to adjust for severity of injury using the duration of LOC, type of TBI, pattern of injury, and underlying comorbidities. Rates were highest in patients with SDH and blunt trauma with closed head injury, which is consistent with those reported in prior literature. ${ }^{18,22}$ The rate of PTS in shaken infant syndrome was strikingly higher than the overall baseline rate ( $41.4 \%$ vs $6.9 \%)$. The rate due to accidental falls $(8.1 \%)$ was elevated as well. The devastating effects of shaken infant syndrome are well described, and neurological sequelae typically occur due to the rupture of bridging veins by shearing forces and subsequent buildup of subdural blood in the subdural potential space. It has been postulated that the hematoma and its subsequent degradation products are irritants to the cortical surface of the brain and are highly epileptogenic. ${ }^{30}$ Thus, injury patterns that do not expose the cortical surface to blood products, such as EDH, were not associated with PTS. In addition, SDHs typically involve worse brain injury than SAHs and EDHs and frequently cause midline shift, LOC, and herniation. The lower rates of PTS in EDH are likely attributable to decreased lucidness during diagnostic workup and decreased detection of seizures via cEEG once LOC develops. Consistent with clinical experience
TABLE 3. Multivariable risk factors for PTS

\begin{tabular}{|c|c|c|}
\hline Risk Factor & OR $(95 \% \mathrm{Cl})$ & p Value \\
\hline \multicolumn{3}{|l|}{ Age group, yrs } \\
\hline $0-5$ & Reference & \\
\hline $6-10$ & $0.6(0.6-0.7)$ & $<0.001$ \\
\hline $11-15$ & $0.7(0.7-0.8)$ & $<0.001$ \\
\hline $16-20$ & $0.5(0.5-0.6)$ & $<0.001$ \\
\hline \multicolumn{3}{|l|}{ Race } \\
\hline White & Reference & \\
\hline African American & $1.4(1.3-1.5)$ & $<0.001$ \\
\hline Hispanic & $1.2(1.1-1.3)$ & $<0.001$ \\
\hline Minority & $1.1(1.0-1.2)$ & 0.10 \\
\hline \multicolumn{3}{|l|}{ No. of comorbidities } \\
\hline 0 & Reference & \\
\hline 1 & $2.0(1.9-2.1)$ & $<0.001$ \\
\hline 2 & $2.9(2.7-3.2)$ & $<0.001$ \\
\hline$\geq 3$ & $4.0(3.6-4.5)$ & $<0.001$ \\
\hline \multicolumn{3}{|l|}{ Etiology of injury (\%) } \\
\hline MVA & Reference & \\
\hline Shaken infant syndrome & $4.4(3.2-5.9)$ & $<0.001$ \\
\hline Fall & $1.3(1.2-1.4)$ & $<0.001$ \\
\hline Firearm & - & - \\
\hline \multicolumn{3}{|l|}{ Type of injury } \\
\hline Concussion & Reference & Reference \\
\hline $\mathrm{SDH}$ & $1.6(1.5-2.3)$ & $<0.001$ \\
\hline $\mathrm{EDH}$ & $0.5(0.4-0.7)$ & $<0.001$ \\
\hline \multicolumn{3}{|l|}{ Severity of injury } \\
\hline No LOC & Reference & Reference \\
\hline Brief LOC (<1 hr) & $1.4(1.3-1.5)$ & $<0.001$ \\
\hline Moderate LOC (1-24 hrs) & $1.5(1.1-2.0)$ & 0.01 \\
\hline $\begin{array}{l}\text { Prolonged LOC ( } \geq 24 \mathrm{hrs}) \text {, baseline } \\
\text { return }\end{array}$ & $1.8(1.4-2.3)$ & $<0.001$ \\
\hline Prolonged LOC ( $\geq 24 \mathrm{hrs}$ ), no return & $0.8(0.7-0.9)$ & 0.01 \\
\hline \multicolumn{3}{|l|}{ Nature of injury } \\
\hline Open & Reference & Reference \\
\hline Closed & $2.3(1.5-3.4)$ & $<0.001$ \\
\hline \multicolumn{3}{|l|}{ cEEG monitoring } \\
\hline No & Reference & Reference \\
\hline Yes & $4.9(3.7-6.4)$ & $<0.001$ \\
\hline \multicolumn{3}{|l|}{ Surgical management } \\
\hline No & Reference & Reference \\
\hline Yes & $1.5(1.3-1.7)$ & $<0.001$ \\
\hline \multicolumn{3}{|l|}{ Evacuation on admission } \\
\hline No & Reference & Reference \\
\hline Yes & $0.8(0.7-0.9)$ & 0.006 \\
\hline \multicolumn{3}{|l|}{ Hospital teaching status } \\
\hline Nonteaching & Reference & Reference \\
\hline Teaching & $1.1(1.05-1.2)$ & 0.001 \\
\hline
\end{tabular}


TABLE 4. In-hospital complications and discharge disposition

\begin{tabular}{|c|c|c|c|}
\hline Outcome Variable & $\begin{array}{c}\text { No. of } \\
\text { Patients }\end{array}$ & $\begin{array}{c}\text { No. w/ PTS } \\
(\%)\end{array}$ & $\begin{array}{c}p \\
\text { Value }\end{array}$ \\
\hline Total no. & 124,444 & $8,597(6.9)$ & \\
\hline \multicolumn{4}{|l|}{ Complication } \\
\hline Any complication & 15,806 & $1,928(12.2)$ & $<0.001$ \\
\hline Neurological & 183 & $28(15.5)$ & $<0.001$ \\
\hline Hemorrhage/hematoma & 170 & $30(17.9)$ & $<0.001$ \\
\hline Thromboembolic & 1,075 & $143(13.1)$ & $<0.001$ \\
\hline Pulmonary & 15,005 & $1,831(12.2)$ & $<0.001$ \\
\hline Cardiac & 70 & $11(16.0)$ & 0.02 \\
\hline Urinary/renal & 501 & $46(9.1)$ & 0.12 \\
\hline Septicemia & 200 & $29(14.4)$ & $<0.001$ \\
\hline Discharge disposition & & & $<0.001$ \\
\hline Routine & 101,597 & $6,604(6.5)$ & \\
\hline Transfer to short-term hospital & 2,851 & $342(12.0)$ & \\
\hline Transfer to SNF or ICF & 10,771 & $197(11.1)$ & \\
\hline Home healthcare & 1,771 & $163(9.2)$ & \\
\hline Against medical advice & 646 & $41(6.3)$ & \\
\hline In-hospital mortality & 6,700 & $288(4.3)$ & \\
\hline
\end{tabular}

ICF = intermediate care facility; SNF = skilled nursing facility.

and prior literature, we found that EDH was associated with a decreased risk of PTS, which may historically be the result of decreased lucidness in these patients and decreased clinical sensitivity for seizures without widespread utilization of cEEG. From clinical experience, $\mathrm{SAH}$, particularly spontaneous $\mathrm{SAH}$, is usually associated with epileptic seizures. We did not find that posttraumatic $\mathrm{SAH}$, which is a very different pathology from spontaneous $\mathrm{SAH}$, was associated with high rates of seizures. Lastly, open injuries were predictive of decreased PTS risk in comparison with closed injuries, which we speculate is the result of decreased intracranial pressure and herniationrelated complications.

\section{In-Hospital Outcomes of PTS}

When examining outcomes, we found that PTS was associated with an increased likelihood of in-hospital complications and adverse discharge but less likelihood of mortality. Thus, the suggestion that PTS is associated with decreased mortality is of unclear significance. More concerning is the association between PTS and adverse discharge, which includes increased placement in nursing homes, long-term care facilities, and home healthcare. This supports prior literature suggesting that early PTS can result in poor recovery from TBI due to hypoxic-ischemic brain injury and development of posttraumatic epilepsy.

\section{Clinical Implications and Future Directions}

Urgent reduction of PTS rates is paramount, which may be achieved by increased utilization of AEDs and risk stratification to identify the patients most likely to benefit from cEEG monitoring and urgent surgical intervention. The highest-risk groups include those with transient to prolonged LOC and closed injury patterns, including SDH and shaken infant syndrome. Currently, our recommendation is that all young patients with a TBI that is more severe than concussion receive at least temporary prophylactic AEDs. Routine cEEG is encouraged if available and may allow for discontinuation of AEDs. The failure to prescribe AEDs to a patient subclinically seizing while in a temporary comatose state is detrimental to cerebral recovery and will likely worsen long-term outcomes, including the rate of posttraumatic epilepsy. A study of prophylactic antiepileptic use in children with severe TBI found that $52 \%$ received prophylactic AEDs. ${ }^{26}$ A study in children with mild TBI found that only $2 \%$ received AEDs prophylactically and < $1 \%$ underwent cEEG..$^{15}$ The impact of antiepileptic agents on long-term seizure outcomes could not be assessed using the KID, which has limited information regarding pharmacotherapy and follow-up visits. The impact of surgical intervention on PTS is less clear and currently controversial.

Analysis of nationwide big data sources such as the KID is very useful for identifying epidemiological trends and associations over time and between variables. Thus, these types of analyses may provide justification for the design of future prospective studies, including multicenter registries, geared at addressing specific topics in neurosurgery. Our results and review of the literature support the design of a well-powered and controlled study comparing the rates of PTS in patients receiving early surgical intervention and medical therapy versus medical therapy alone. Considering the heterogeneity of the pediatric TBI patient population, we would encourage stratified analysis and application of risk stratification to identify which groups, if any, benefit most from surgery. Our results suggest a low rate of nationwide cEEG monitoring and the possibility of undetected PTSs in patients who have temporarily lost consciousness. A future study is advised to investigate the effects of cEEG on the long-term neurological outcomes, including persistent epilepsy, in children admitted with PTS.

\section{Limitations}

Despite its many strengths, the KID has many limitations inherent to a large database system. One of the major limitations is the subjectivity of the ICD-9-CM code used to classify these patients. Health professionals from different locations may have varying coding procedures based on incorrectly entered codes or environmental and societal imbalances. Such variability reflects the sensitivity and sometimes specificity of the coding process. In the context of our data, variability can arise in the documentation of the duration of LOC and severity of TBI. However, the differentiation in this study by severity and LOC is still very important and is one of the study's greatest attributes. Any variability that arises in these categories is not significant enough to create measurably biased results (due to the size of the cohort, variability would be minimized and be evenly spread through all sections of the cohort). While the KID holds a vast amount of data, not all 50 states contribute to the database. Therefore, there may be additional bias presented in the results due to the lack of patient data from certain regions of the nation. Presently, there is no way to minimize bias from this source. In addition, all the ICD-9-CM codes are records from hospital stays.

Several limitations may have underestimated the true 
incidence of PTS in our study, including the lack of clinical detection in severe TBI patients with LOC or coma, lack of readmissions data, and lack of outpatient follow-up data. The ICD-9-CM coding system does not have the specificity to distinguish between PTS and epilepsy and thus we cannot determine how many patients continued to have persistent seizures at long-term follow-up. The KID does not contain details regarding pharmacotherapy, prohibiting our ability to assess the efficacy of prophylactic antiepileptic agents. However, clinical experience suggests that it is standard of care and relatively commonplace to administer prophylactic AEDs to all PTS patients. We refrained from evaluating the efficacy of cEEG on outcomes due to the lack of clinically useful outcome measures available in our database. Important outcomes would include seizure freedom at 6 months as well as overall neurological disability. The inability of KID variables to completely and accurately control for severity of TBI limits the generalizability of an outcomes analysis of predictors of mortality. Despite the limitations provided primarily by the database in this study, we still believe that the results provide novel information to health professionals and patients that could not be derived from traditional single-center cohort studies.

\section{Conclusions}

In this study, we utilized the largest pediatric inpatient database in the US to report the first national incidence of PTS in TBI patients. In this cohort, we identified independent risk factors for PTS, which included younger age $(<$ 5 years), African American race, preexisting comorbidity, prolonged loss of consciousness, and injury pattern involving cortical exposure to blood products. However, we also identified factors that decreased the likelihood of PTS, such as early surgical evacuation. Many of the risk factors were consistent with those in prior clinical studies or explained by pathophysiological explanations from basic science literature. However, further prospective clinical studies will be necessary to confirm and attempt to modify the factors identified in our study.

\section{References}

1. Agrawal A, Timothy J, Pandit L, Manju M: Post-traumatic epilepsy: an overview. Clin Neurol Neurosurg 108:433-439, 2006

2. Ahmed S, Venigalla H, Mekala HM, Dar S, Hassan M, Ayub S: Traumatic brain injury and neuropsychiatric complications. Indian J Psychol Med 39:114-121, 2017

3. Annegers JF, Hauser WA, Coan SP, Rocca WA: A population-based study of seizures after traumatic brain injuries. $\mathbf{N}$ Engl J Med 338:20-24, 1998

4. Arango JI, Deibert CP, Brown D, Bell M, Dvorchik I, Adelson PD: Posttraumatic seizures in children with severe traumatic brain injury. Childs Nerv Syst 28:1925-1929, 2012

5. Ateş O, Ondüi S, Onal C, Büyükkiraz M, Somay H, Cayli SR, et al: Post-traumatic early epilepsy in pediatric age group with emphasis on influential factors. Childs Nerv Syst 22:279-284, 2006

6. Bakr A, Belli A: A systemic review of levetiracetam versus phenytoin in the prevention of late post-traumatic seizures and survey of UK neurosurgical prescribing practice of antiepileptic medication in acute traumatic brain injury. Br J Neurosurg [epub ahead of print], 2018
7. Chang BS, Lowenstein DH: Practice parameter: antiepileptic drug prophylaxis in severe traumatic brain injury: report of the Quality Standards Subcommittee of the American Academy of Neurology. Neurology 60:10-16, 2003

8. Chen H, Yuan F, Chen SW, Guo Y, Wang G, Deng ZF, et al: Predicting posttraumatic hydrocephalus: derivation and validation of a risk scoring system based on clinical characteristics. Metab Brain Dis 32:1427-1435, 2017

9. Dingman AL, Stence NV, O’Neill BR, Sillau SH, Chapman KE: Seizure severity is correlated with severity of hypoxicischemic injury in abusive head trauma. Pediatr Neurol 82:29-35, 2018

10. Elixhauser A, Steiner C, Harris DR, Coffey RM: Comorbidity measures for use with administrative data. Med Care 36:8-27, 1998

11. Englander J, Bushnik T, Duong TT, Cifu DX, Zafonte R, Wright J, et al: Analyzing risk factors for late posttraumatic seizures: a prospective, multicenter investigation. Arch Phys Med Rehabil 84:365-373, 2003

12. Hale AT, Pekala K, Theobald B, Kelly K, Wolf M, Wellons JC, et al: Predictors of post-discharge seizures in children with traumatic brain injury. Childs Nerv Syst 34:1361-1365, 2018

13. HCUP: Clinical Classifications Software (CCS) for ICD-9CM. Rockville, MD: Agency for Healthcare Research and Quality, 2011. (https://www.hcup-us.ahrq.gov/toolssoftware/ ccs/ccs.jsp) [Accessed July 30, 2018]

14. HCUP: Overview of the Kids' Inpatient Database (KID). Rockville, MD: Agency for Healthcare Research and Quality (AHRQ), 2003. (https://www.hcup-us.ahrq.gov/kidoverview. jsp) [Accessed July 30, 2018]

15. Huguenard AL, Miller BA, Sarda S, Capasse M, Reisner A, Chern JJ: Mild traumatic brain injury in children is associated with a low risk for posttraumatic seizures. J Neurosurg Pediatr 17:476-482, 2016

16. Kilbride RD, Costello DJ, Chiappa KH: How seizure detection by continuous electroencephalographic monitoring affects the prescribing of antiepileptic medications. Arch Neurol 66:723-728, 2009

17. Kuluz J: Posttraumatic seizures in children with severe traumatic brain injury. Pediatr Crit Care Med 18:87-88, 2017

18. Liesemer K, Bratton SL, Zebrack CM, Brockmeyer D, Statler KD: Early post-traumatic seizures in moderate to severe pediatric traumatic brain injury: rates, risk factors, and clinical features. J Neurotrauma 28:755-762, 2011

19. Mazzini L, Campini R, Angelino E, Rognone F, Pastore I, Oliveri G: Posttraumatic hydrocephalus: a clinical, neuroradiologic, and neuropsychologic assessment of long-term outcome. Arch Phys Med Rehabil 84:1637-1641, 2003

20. Omar M, Moore L, Lauzier F, Tardif PA, Dufresne P, Boutin A, et al: Complications following hospital admission for traumatic brain injury: a multicenter cohort study. J Crit Care 41:1-8, 2017

21. O'Neill BR, Handler MH, Tong S, Chapman KE: Incidence of seizures on continuous EEG monitoring following traumatic brain injury in children. J Neurosurg Pediatr 16:167176, 2015

22. Petridis AK, Doukas A, Maslehaty H, Mehdorn HM: Predictors and incidence of posttraumatic seizures in children and adolescents after brain injury. Clin Pract 2:e66, 2012

23. Ratan SK, Kulshreshtha R, Pandey RM: Predictors of posttraumatic convulsions in head-injured children. Pediatr Neurosurg 30:127-131, 1999

24. Ritter AC, Wagner AK, Fabio A, Pugh MJ, Walker WC, Szaflarski JP, et al: Incidence and risk factors of posttraumatic seizures following traumatic brain injury: a Traumatic Brain Injury Model Systems study. Epilepsia 57:1968-1977, 2016

25. Rumalla K, Letchuman V, Smith KA, Arnold PM: Hydrocephalus in pediatric traumatic brain injury: national inci- 
dence, risk factors, and outcomes in 124,444 hospitalized patients. Pediatr Neurol 80:70-76, 2018

26. Ruzas CM, DeWitt PE, Bennett KS, Chapman KE, Harlaar N, Bennett TD: EEG monitoring and antiepileptic drugs in children with severe TBI. Neurocrit Care 26:256-266, 2017

27. Szaflarski JP, Nazzal Y, Dreer LE: Post-traumatic epilepsy: current and emerging treatment options. Neuropsychiatr Dis Treat 10:1469-1477, 2014

28. Temkin NR: Preventing and treating posttraumatic seizures: the human experience. Epilepsia 50 (Suppl 2):10-13, 2009

29. Weintraub AH, Gerber DJ, Kowalski RG: Posttraumatic hydrocephalus as a confounding influence on brain injury rehabilitation: incidence, clinical characteristics, and outcomes. Arch Phys Med Rehabil 98:312-319, 2017

30. Won SY, Konczalla J, Dubinski D, Cattani A, Cuca C, Seifert $\mathrm{V}$, et al: A systematic review of epileptic seizures in adults with subdural haematomas. Seizure 45:28-35, 2017

\section{Disclosures}

Dr. Paul Arnold has commercial relationships with Z-Plasty (stock, stock options, or other ownership interest); Medtronic Sofamor Danek (salary and any payment for services not otherwise identified as salary, such as consulting, fees, honoraria, paid authorship, or other payments for services); Stryker Spine (sponsored or reimbursed travel, salary and any payment for services not otherwise identified as salary, such as consulting, fees, honoraria, paid authorship, or other payments for services); AOSpine North America (sponsored or reimbursed travel); Invivo (salary and any payment for services not otherwise identified as salary, such as consulting, fees, honoraria, paid authorship, or other payments for services); SpineEx (shares in company); and Asterias (financial, data and safety monitoring board); and noncommercial relationships with Lumbar Spine Research Society (nonfinancial, co-chair of Program Committee); AANS/CNS Joint Section Neurotrauma (executive committee); and CSRS (nonfinancial, board of directors).

\section{Author Contributions}

Conception and design: Rumalla. Acquisition of data: Rumalla. Analysis and interpretation of data: Rumalla, Smith, Letchuman. Drafting the article: Rumalla, Letchuman, Gandham, Kombathula. Critically revising the article: Arnold, Rumalla, Smith, Letchuman. Reviewed submitted version of manuscript: Arnold, Rumalla, Smith. Administrative/technical/material support: Arnold, Smith. Study supervision: Arnold.

\section{Correspondence}

Paul M. Arnold: University of Kansas Medical Center, Kansas City, KS.parnold@kumc.edu. 